\title{
MERCADO DE TRABALHO EM SAÚDE NO BRASIL: EMPREGOS PARA OS ENFERMEIROS NAS TRÊS ÚLTIMAS DÉCADAS
}

\author{
THE HEALTH CARE LABOR MARKET IN BRAZIL: EMPLOYMENT FOR \\ NURSING PROFESSIONALS IN THE LAST THREE DECADES \\ EL MERCADO DE TRABAJO EN SALUD EN BRASIL: EMPLEOS DE LOS \\ ENFERMEROS EN LAS TRES ÚLTIMAS DÉCADAS
}

Ana Luiza Stiebler Vieira'

Eliane dos Santos Oliveira ${ }^{2}$

\begin{abstract}
RESUMO: 0 trabalho mostra a dinâmica dos empregos nas décadas de 70,80 e 90 delineando as principais caracteristicas e tendências do mercado de trabalho dos enfermeiros no Brasil, principalmente antes e após a implantação do Sistema Único de Saúde-SUS. Utiliza como fonte principal os dados divulgados pela pesquisa de Assistência Médica-Sanitária -AMS desenvolvida pelo IBGE.
\end{abstract}

PALAVRAS-CHAVE: enfermeiro, mercado de trabalho, emprego para enfermeiros

\section{INTRODUÇÃO}

A saúde constitui um setor dinâmico no mercado de trabalho em geral que absorve importante parcela da população economicamente ativa do pais. Como os demais serviços de consumo coletivo, a saúde, associada a uma rede de determinantes políticos e econômicos, operou um grande crescimento do seu sistema produtor de serviços e, conseqüentemente, provocou mudanças na estrutura ocupacional do setor, ou seja, tanto no perfil quantitativo quanto no qualitativo da força de trabalho em saúde.

O dinamismo deste mercado de trabalho se revela com a significativa expansão da oferta de empregos em saúde. Em dezenove anos (de 1980 a 1999), houve um incremento bruto de $170,2 \%$ dos postos de trabalho do setor; qual seja, de 573.629 para 1.549 .838 empregos para os profissionais de saúde (IBGE,1980, p. 30-37, 1999b).

Tendo em vista os dados já disponiveis e os recentemente divulgados pela Pesquisa Assistência Médica Sanitária de 1999, pode-se assim traçar as principais tendências do mercado de trabalho para os enfermeiros e sua evolução ao longo das três últimas décadas e ainda, dar continuidade ao tema que constitui um dos objetos do Núcleo de Estudos e Pesquisas de Recursos Humanos em Saúde da Escola Nacional de Saúde Pública/ Fiocruz.

Optou-se por três momentos distintos: o ano de 1978 para caracterizar a década de 70; o ano de 1984 para representar o periodo que antecedeu a reforma do sistema de saúde; e os anos de 1992 e 1999 para visualizar os impactos da implantação do Sistema Único de Saúde e sua consolidação.

' Doutora em Enfermagem, Mestre em Saúde Coletiva, Pesquisadora Associada do Núcleo de Estudos e Pesquisas em Recursos Humanos em Saúde - NERHUS do Departamento de Administração e Planejamento em Saúde - DAPS/ENSP/FIOCRUZ

${ }^{2}$ Mestre em Saúde Pública, Pesquisadora colaboradora do Núcleo de Estudos e Pesquisas em Recursos Humanos em Saúde - NERHUS do Departamento de Administração e Planejamento em Saúde - DAPS/ENSP/FIOCRUZ 


\section{MERCADO DE TRABALHO PARA OS ENFERMEIROS}

Os empregos para enfermeiros tiveram um incremento bruto de $435,5 \%$ ao longo das três últimas décadas. Os dados da tabela 1, mostram que de 1978 a 1999 os postos de trabalho passaram de 13.104 para 70.175 . Observa-se que a expansão ocorreu mais acentuadamente no periodo de 1992 a 1999. Entretanto, os empregos para enfermeiros ainda ocupam uma pequena fatia no mercado de trabalho em saúde. Em 1999, apenas 4,5\% do total de empregos eram ocupados pelos enfermeiros (IBGE,1999ª p. 56, 1999b). Pode-se dizer que implantação do SUS ainda não contribuiu para uma melhor distribuição interna dos profissionais dentro da equipe de saúde pois o mercado de trabalho continua bipolarizado pelos médicos e auxiliares de enfermagem, categorias que detém juntas $49,5 \%$ do total dos empregos do setor (IBGE, 1999a, p. 56, 1999b).

Regionalmente, constata-se pelos dados da Tabela 1 que o Sudeste manteve-se ao longo dos anos, detendo mais de $50 \%$ dos empregos para enfermeiros, tornando-se o grande pólo de concentração de postos de trabalho para esta categoria. Entretanto, já se identifica uma tendência de ampliação de postos de trabalho em outras regiões, principalmente para o Nordeste que elevou sua participação de $18,3 \%$ para $24,8 \%$ e para o Norte que além de ter expandido o seu mercado de trabalho para enfermeiros, aumentou a absorção de $3,1 \%$ para $4,8 \%$ destes profissionais. No Centro-Oeste, embora em números absolutos os postos de trabalho tenham aumentado, a tendência tem sido de decréscimo da sua participação percentual na oferta de empregos para os enfermeiros no pais.

\section{TABELA 1-EMPREGOS DE ENFERMEIROS POR GRANDES REGIÕES SEGUNDO ANOS} SELECIONADOS

\begin{tabular}{|c|c|c|c|c|c|c|c|c|c|c|c|c|}
\hline & \multicolumn{2}{|c|}{ Brasil } & \multicolumn{2}{|c|}{ Norte } & \multicolumn{2}{|c|}{ Nordeste } & \multicolumn{2}{|c|}{ Sudeste } & \multicolumn{2}{|c|}{ Sul } & \multicolumn{2}{|c|}{ C.Oeste } \\
\hline & V.abs & $(\%)$ & V.abs & $(\%)$ & V.abs & $(\%)$ & V.abs & $(\%)$ & V.abs & $(\%)$ & V.abs & $(\%)$ \\
\hline 1978 & 13.104 & 100,0 & 413 & 3,1 & 2394 & 18,3 & 7.857 & 60,0 & 1538 & 11,7 & 902 & 6,9 \\
\hline 1984 & 21.766 & 100,0 & 947 & 4,4 & 4593 & 21,1 & 12.022 & 55,2 & 2677 & 12,3 & 1527 & 7,0 \\
\hline 1992 & 41.501 & 100,0 & 1700 & 4,1 & 9654 & 23,3 & 22.102 & 53,3 & 5795 & 13,9 & 2250 & 5,4 \\
\hline 1999 & 70.175 & 100,0 & 3408 & 4,8 & 17.383 & 24,8 & 35.838 & 51,1 & 9988 & 14,2 & 3558 & 5,1 \\
\hline
\end{tabular}

Fonte: IBGE

Uma caracteristica já histórica, constitui a concentração do mercado de trabalho dos enfermeiros em hospitais. Assim, em 1999, do total de 70.175 empregos no pais, $68,8 \%$ eram ofertados pelas instituições de saúde com internação (IBGE,1999b). Em relação ao total de empregos de enfermeiros em cada região, este percentual era de $73,3 \%$ no Sudeste; $69,4 \%$ no Centro-Oeste; $65,0 \%$ no Sul; de 63,55 no Nordeste e de $59,4 \%$ no Norte (IBGE,1999b)

Historicamente também,o enfermeiro constitui um trabalhador público da saúde já que este setor ao longo das três décadas mantêm a oferta de mais de $60,0 \%$ dos postos de trabalho para estes profissionais (Tabela 2). Considerando que em 1999 (IBGE,1999a , p. 48), 41,3\% dos estabelecimentos de saúde eram de natureza privada, este setor não absorve os enfermeiros com a mesma dinâmica do público, indicando uma maior utilização das outras categorias de enfermagem como força de trabalho desta equipe. Se os estabelecimentos privados tivessem a mesma empregabilidade dos públicos, a oferta em 1999, deveria ter crescido mais 4.914 postos de trabalho para os enfermeiros no pais. Observando ainda que em 1999 (IBGE,1999a, p. 53), $70,0 \%$ do total dos leitos hospitalares disponiveis no pais (484.945 leitos) localizavam-se nas instituições privadas, seria esperado um aumento na oferta de postos de trabalho para os enfermeiros, ainda mais se o setor optasse por uma melhor qualidade da assistência de 
enfermagem hospitalar através da relação profissional/leito.

Interessante entretanto, é notar que a absorção dos enfermeiros em 1999, apresenta praticamente as mesmas proporções do final da década de 70 , ou seja, um pouco mais de $62,0 \%$ e $37,0 \%$ respectivamente nos setores público e privado. Assim, os estabelecimentos privados de saúde voltam a ofertar mais empregos para os enfermeiros na década de 90 e os públicos, embora seja o maior mercado destes profissionais, diminuem a oferta dos postos de trabalho em relação à década de 80 e início dos anos 90 .

TABELA 2 - EMPREGOS DE ENFERMEIROS POR ESFERAS ADMINISTRATIVAS SEGUNDO ANOS SELECIONADOS

\begin{tabular}{l|r|r|r|r|r|r}
\hline & \multicolumn{2}{|c|}{ Total } & \multicolumn{2}{c|}{ Empregos Públicos } & \multicolumn{2}{c}{ Empregos Privados } \\
\hline & V.abs & $(\%)$ & V.abs & $(\%)$ & V.abs & $(\%)$ \\
\hline 1978 & 13.104 & 100,0 & 8.199 & 62,6 & 4.905 & 37,4 \\
\hline 1984 & 21.766 & 100,0 & 14.702 & 67,5 & 7.065 & 32,5 \\
\hline 1992 & 41.501 & 100,0 & 27.081 & 65,3 & 14.420 & 34,7 \\
\hline 1999 & 70.175 & 100,0 & 44.077 & 62,8 & 26.098 & 37,2 \\
\hline
\end{tabular}

Fonte: IBGE

Entre todas as mudanças no mercado de trabalho em saúde para os enfermeiros no pais nas três últimas décadas, a mais significativa encontra-se na municipalização da oferta dos empregos (Tabela 3). Os municipios em 1999 passaram a ofertar $34,6 \%$ (24.281 empregos) do total dos postos de trabalho para os enfermeiros em todo o pais (70.175), o que equivale praticamente a toda oferta do setor privado também no pais (de $37,2 \%$ ou 26.098 empregos). Em relação ao total dos empregos públicos em 1999 (44.077), os municipios se tornaram os maiores empregadores de enfermeiros, ofertando $55,1 \%$ destes postos de trabalho.

Até a década de 80 , a esfera pública federal constituia o maior mercado de trabalho dos enfermeiros; na década de 90, o quadro se reverte. Em 1999, os empregos públicos federais passaram a representar apenas $8,8 \%$ do total de empregos no setor público (em 1978 era de $46,2 \%$ do total dos postos de trabalho para os enfermeiros).

Esta grande mudança no mercado de trabalho dos enfermeiros verificada nos anos 90 , igualmente ocorreu em todo o mercado de trabalho em saúde (IBGE, 1999b) como resultado da efetiva implantação da descentralização do sistema de saúde a qual revela também a descentralização da demanda por enfermeiros e profissionais de saúde no pais para o âmbito dos municipios.

TABELA 3 - EMPREGOS PÚBLICOS DE ENFERMEIROS POR NIVEIS ADMINISTRATIVOS SEGUNDO ANOS SELECIONADOS

\begin{tabular}{l|r|r|r|r|r|r}
\hline & \multicolumn{2}{|c|}{ Federal } & \multicolumn{2}{c|}{ Estadual } & \multicolumn{2}{c}{ Municipal } \\
\hline & V.abs & $(\%)$ & V.abs & $(\%)$ & V.abs & $(\%)$ \\
\hline 1978 & 3.792 & 29,0 & 3.214 & 24,5 & 1.193 & 9,1 \\
\hline 1984 & 6.357 & 29,2 & 6.294 & 28,9 & 2.051 & 9,4 \\
\hline 1992 & 4.984 & 12,0 & 12.390 & 29,9 & 9.707 & 23,4 \\
\hline 1999 & 6.191 & 8,8 & 13.605 & 19,4 & 24.281 & 34,6 \\
\hline
\end{tabular}

Fonte: IBGE

A última pesquisa sobre mercado de trabalho em saúde (IBGE, 1999), contemplou duas temáticas importantes para análise deste mercado: a jornada de trabalho e as formas de vínculo 
dos profissionais nas instituições. Classificou como jornada de trabalho integral, aquela de carga horária minima de 40 horas semanais; parcial, de carga minima inferior a 40 horas semanais; e indefinido, de carga horária variável. Como formas de vinculo, considerou o próprio, em que o contrato é feito direto com o estabelecimento de saúde; intermediário em que o contrato é através de empresa, cooperativa ou outro tipo de entidade diferente do estabelecimento; e outro, através de prestador de serviço e autônomo.

Embora prevaleça no pais a vinculação própria (Tabelas 4 e 5), pode-se claramente observar indicios de flexibilização do mercado de trabalho dos enfermeiros através dos contratos por cooperativas ou empresas (intermediários) e da prestação de serviços e de autônomos nos estabelecimentos de saúde. Somados, estes vinculos, já representavam em 1999 (Tabela 4), $11,3 \%$ dos empregos para os enfermeiros no Brasil sendo mais expressivos no setor público $(12,6 \%)$, que no setor privado $(9,0 \%)$.

Tendo em vista que o setor privado emprega $91,0 \%$ dos enfermeiros através de contratação própria e que o setor público vem utilizando com maior freqüência a contratação intermediária, a prestação de serviços e de autônomos nos seus estabelecimentos, pode-se dizer que a flexibilização do mercado de trabalho para os enfermeiros vem sendo mais dinâmica no setor público, o qual corresponde ao seu maior mercado de trabalho no pais. Ao observarmos nos niveis administrativos (Tabela 5), $12,4 \%$ dos empregos federais; $11,4 \%$ dos estaduais e $13,4 \%$ dos empregos municipais têm vinculação intermediária e outro (prestação de serviços e de autônomos nos seus estabelecimentos).

Como o mercado de trabalho em saúde constitui um mercado interdependente do mercado de trabalho em geral e das politicas governamentais, estas vinculaçöes de trabalho do enfermeiro no setor público espelham a politica de reforma administrativa do Estado e a politica de emprego no pais.

Na descentralização dos mecanismos de gestão do SUS, o sistema de "gestão plena" confere maior grau de autonomia ao gestor local. Assim, para enfrentar a rigidez da norma estatutária para contratação de profissionais, a solução encontrada pelo gestor local passa, segundo Nogueira (1999, p. 7), por algum tipo de terceirização da força de trabalho em saúde. Como afirma o autor, as terceirizações envolvem uma mediação feita por entidade privada (empresa, ONG, ou cooperativa) que conta, em comparação com a administração pública, com maior liberdade para contratação e fixação de niveis de remuneração.

Em muitos casos, principalmente no Programa de Saúde da Familia, os diferentes contratos constituem em incentivo à dedicação através de remuneração mais elevada do que as ofertadas em hospitais e ambulatórios.

Entretanto, estas formas de vinculação com os serviços de saúde, apesar de ágeis sob o ponto de vista gerencial, apresentam desvantagens bastante relevantes como a perda de direitos trabalhistas, a rotatividade de profissionais, o incentivo ao descompromisso com a instituição e com a qualidade da assistência.

Nas cooperativas, onde além de apresentar as desvantagens citadas, já não é incomum o atraso dos salários, a utilização de profissionais cadastrados como free-lances para cobrir plantões em dias, setores e até em diferentes hospitais e ainda, a utilização de mão-de-obra gratuita através de "plantões teste" em setores e plantões descobertos, como uma etapa de seleção para o ingresso do enfermeiro na organização.

Como pode ser visualizado também nas tabelas 4 e 5, em 1999, a jornada integral prevalece no mercado de trabalho dos enfermeiros no pais, no setor público e privado. No entanto, já se torna bastante expressiva a jornada parcial principalmente no setor público (Tabela 5), notadamente na esfera municipal, a qual pode ter sido impulsionada pela contratação intermediária. Na esfera estadual e principalmente na federal, predomina a jornada de trabalho integral. 
TABELA 4- TOTAL DE EMPREGOS DE ENFERMEIROS POR ESFERAS ADMINISTRATIVAS SEGUNDO FORMAS DE VÍNCULOE JORNADA DE TRABALHO, BRASIL1999

\begin{tabular}{|c|c|c|c|c|c|c|}
\hline \multirow{2}{*}{$\begin{array}{c}\text { Formas de Vínculos } \\
\text { de Trabalho }\end{array}$} & \multicolumn{2}{|c|}{ Total } & \multicolumn{2}{|c|}{ Público } & \multicolumn{2}{|c|}{ Privado } \\
\hline & V.abs & $(\%)$ & V.abs & $(\%)$ & V.abs & $(\%)$ \\
\hline Próprio & 62.251 & 88,7 & 38.501 & 87,4 & 23.750 & 91,0 \\
\hline Intermediário & 4.618 & 6,6 & 3.501 & 7,9 & 1.117 & 4,3 \\
\hline Outro & 3.306 & 4,7 & 2.075 & 4,7 & 1.231 & 4,7 \\
\hline Total & 70.175 & 100,0 & 44.077 & 100,0 & 26.098 & 100,0 \\
\hline Jornada de Trabalho & \multicolumn{2}{|c|}{ Total } & \multicolumn{2}{|c|}{ Público } & \multicolumn{2}{|c|}{ Privado } \\
\hline & V.abs & $(\%)$ & V.abs & $(\%)$ & V.abs & $(\%)$ \\
\hline Integral & 38.426 & 54,8 & 23.046 & 52,2 & 15.380 & 58,9 \\
\hline Parcial & 30.367 & 43,3 & 20.343 & 46,2 & 10.024 & 38,4 \\
\hline Indefinido & 1.382 & 1,9 & 688 & 1,6 & 694 & 2,7 \\
\hline Total & 70.175 & 100,0 & 44.077 & 100,0 & 26.098 & 100,0 \\
\hline
\end{tabular}

Fonte: IBGE

TABELA 5-EMPREGOS PÚBLICOS DE ENFERMEIROS POR NIVEIS ADMINISTRATIVOS SEGUNDO FORMAS DE VÍNCULO E JORNADA DE TRABALHO, BRASIL-1999

\begin{tabular}{|c|c|c|c|c|c|c|c|c|}
\hline \multirow[t]{2}{*}{$\begin{array}{c}\text { Formas de Vínculos } \\
\text { de Trabalho }\end{array}$} & \multicolumn{2}{|c|}{$\begin{array}{l}\text { Total de } \\
\text { Empregos } \\
\text { Públicos }\end{array}$} & \multicolumn{2}{|c|}{ Federal } & \multicolumn{2}{|c|}{ Estadual } & \multicolumn{2}{|c|}{ Municipal } \\
\hline & V.abs & (\%) & V.abs & (\%) & V.abs & $(\%)$ & V.abs & $(\%)$ \\
\hline Próprio & 38501 & 87,3 & 5425 & 87,6 & 12.049 & 88,6 & 21.027 & 86,6 \\
\hline Intermediário & 3501 & 7,9 & 520 & 8,4 & 883 & 6,5 & 2.098 & 8,6 \\
\hline Outro & 2075 & 4,8 & 246 & 4,0 & 673 & 4,9 & 1.156 & 4,8 \\
\hline Total & 44.077 & 100,0 & 6.191 & 100,0 & 13.605 & 100,0 & 24.281 & 100,0 \\
\hline \multirow[t]{2}{*}{ Jornada de Trabalho } & \multicolumn{2}{|c|}{$\begin{array}{l}\text { Total de } \\
\text { Empregos } \\
\text { Públicos }\end{array}$} & \multicolumn{2}{|c|}{ Federal } & \multicolumn{2}{|c|}{ Estadual } & \multicolumn{2}{|c|}{ Municipal } \\
\hline & V.abs & $(\%)$ & V.abs & $(\%)$ & V.abs & $(\%)$ & V.abs & $(\%)$ \\
\hline Integral & 23.046 & 52,3 & 4.464 & 72,1 & 7.536 & 55,4 & 11.046 & 45,5 \\
\hline Parcial & 20.343 & 46,1 & 1.663 & 26,9 & 5.887 & 43,3 & 12.793 & 52,7 \\
\hline Indefinido & 688 & 1,6 & 64 & 1,0 & 182 & 1,3 & 442 & 1,8 \\
\hline Total & 44.077 & 100,0 & 6.191 & 100,0 & 13.605 & 100,0 & 24.281 & 100,0 \\
\hline
\end{tabular}

Fonte: IBGE

\section{CONSIDERAÇÕES FINAIS}

Levando em consideração a análise da distribuição de empregos para enfermeiros no mercado de trabalho em saúde no pais, podemos apontar as principais tendências deste mercado nas três últimas décadas:

- Crescimento significativo da oferta de empregos ou da demanda por enfermeiros no sistema de saúde;

- Desconcentração da oferta de postos de trabalho da região Sudeste de forma contínua ainda que a região permaneça concentrando mais de $50,0 \%$ da oferta de empregos para os enfermeiros;

- Crescimento significativo do mercado de trabalho dos enfermeiros na região Nordeste;

- Concentração histórica de postos de trabalho para os enfermeiros nos hospitais que 
também constitui ainda o seu maior mercado de trabalho;

- Manutenção da histórica inserção dos enfermeiros no mercado público de saúde embora na década de 90 se observe um ligeiro aumento da oferta de empregos no setor privado;

- O setor privado de saúde constitui ainda um mercado de trabalho restrito para os enfermeiros considerando a sua capacidade instalada;

-Desfederalização significativa da demanda por enfermeiros e municipalização acentuada do mercado de trabalho para os enfermeiros;

- Jornada de trabalho que praticamente se divide entre integral e parcial. Mas no mercado privado o enfermeiro trabalha majoritariamente em tempo integral enquanto que nos municipios, há a preponderância do trabalho em tempo parcial;

- Indicios de flexibilização do mercado de trabalho dos enfermeiros através de contratos por empresas, cooperativas, da prestação de serviços e de trabalho autônomo destes profissionais nas instituições de saúde;

- Maior dinâmica de flexibilização do mercado de trabalho dos enfermeiros no setor público que constitui o seu grande empregador;

- Municipalização aliada à tendência de flexibilização deste mercado através da precarização contratual dos enfermeiros nas instituições municipais de saúde.

Embora tenha-se observado o mercado de trabalho para os enfermeiros no aspecto quantitativo, estes dados são suficientes para apontar mudanças importantes da sua inserção nas instituições de saúde. O mercado de trabalho de fato, vem sofrendo reestruturações preocupantes entre elas a sua flexibilização, através de contratos precários com perda de direitos trabalhistas, os quais comprometem a cidadania dos profissionais de saúde e em especial dos enfermeiros.

ABSTRACT: The present study shows the dynamics of employment for the nursing profession in Brazil from 1970 up to 1990. It outlines the main characteristics and trends of this labor market before and after the implantation of the Sistema Único de Saúde-SUS (Federal Health Care System). The main source of information for this investigation was data published in the research Medical-Sanitary Assistance carried out by IBGE (Brazilian Geography and Statistics Institute).

KEYWORDS: nurse, works market, nurse's employments

RESUMEN: El trabajp muestra la dinámica de los empleos en las décadas de 70,80 y 90 delineando las principales caracteristicas y tendencias del mercado de trabajo de los enfermeros en Brasil, principalmente antes y después de la implantación del Sistema Único de Salud-SUS. Se utiliza como fuente principal los datos divulgados por la investigación de Asistencia Medico- Sanitaria-AMS Ilevada a cabo por el IBGE.

PALABRAS CLAVE: enfermero, mercado de trabajo, empleo para enfermeros

\section{REFERÊNCIAS BIBLIOGRÁFICAS}

IBGE. Estatísticas da Saúde: Assistência Médica Sanitária. Rio de Janeiro, IBGE, 1978. v.3, 70 p.

IBGE. Estatísticas da Saúde: Assistência Médica Sanitária. Rio de Janeiro, IBGE, 1980. v.5, 56 p.

IBGE. Estatisticas da Saúde: Assistência Médica Sanitária. Rio de Janeiro. IBGE, 1986. v.9, 66 p. 
IBGE. Estatisticas da Saúde: Assistência Médica Sanitária. Rio de Janeiro, 1992. v.17, 155 p.

IBGE. 1999a. Estatísticas da Saúde: Assistência Médica Sanitária. Rio de Janeiro. IBGE, 2000. 106p.

IBGE. 1999b. Estatisticas da Saúde: Assistência Médica Sanitária. Tabulações Especiais. Ministério da Saúde. Secretaria Executiva. Datasus, 2:000.

NOGUEIRA, R. P. A regulação dos vinculos de trabalho no SUS. Brasilia, 1999. 28p. Mimeogr. 